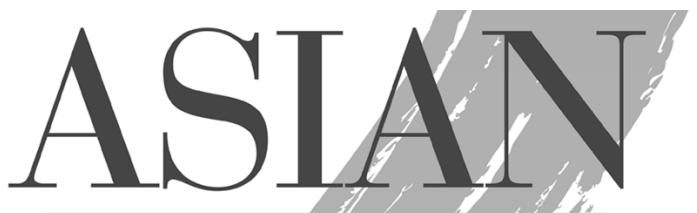

CARDIOVASCULAR \& THORACIC

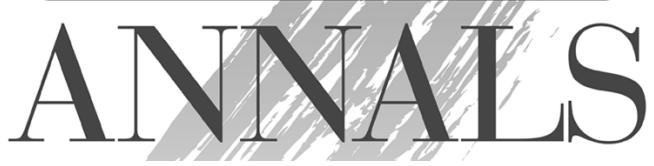

Solitary Fibrous Tumor of the Pleura: Surgery and Clinical Course in 18 Cases Alessandro Bini, Jury Brandolini, Fabio Davoli, Giampiero Dolci, Francesco Sellitri and Franco Stella

Asian Cardiovasc Thorac Ann 2009;17:378-381

DOI: $10.1177 / 0218492309338103$

This information is current as of December 27, 2011

The online version of this article, along with updated information and services, is located on the World Wide Web at:

http://asianannals.ctsnetjournals.org/cgi/content/full/17/4/378

The Asian Cardiovascular \& Thoracic Annals is the official journal of The Asian Society for Cardiovascular Surgery and affiliated journal of The Association of Thoracic and Cardiovascular Surgeons of Asia. 


\title{
Solitary Fibrous Tumor of the Pleura: Surgery and Clinical Course in 18 Cases
}

\author{
Alessandro Bini, MD, Jury Brandolini, MD, Fabio Davoli, MD, \\ Giampiero Dolci, MD, Francesco Sellitri, MD, Franco Stella, MD
}

Department of Thoracic Surgery

University of Bologna, S. Orsola-Malpighi Hospital

Bologna, Italy

\begin{abstract}
Solitary fibrous tumors of the pleura are very rare neoplasms that can sometimes present with malignant features. Between 1984 and 2007, 18 cases were treated in our institution. There were 7 men and 11 women, with a median age of 56 years (range, 33-77 years). All patients underwent surgical treatment. Except for one case with hemangiopericytic features, all tumors were histologically the fibrous type of pleural mesothelioma. Resections were radical and there were no recurrences. There was no perioperative mortality. The outcome was excellent, and all patients have been followed up continuously. Survival rates at 3, 5, and 10 years were calculated as $86.7 \%, 75 \%$, and $66.7 \%$. One patient died after 18 months (malignant type of solitary fibrous tumor), and 2 died of unrelated disease after 24 and 53 months. Surgery is the treatment of choice, and careful long-term clinical follow-up is required.
\end{abstract}

(Asian Cardiovasc Thorac Ann 2009;17:378-81)

KEYWORDS: Pleura, Solitary Fibrous Tumor, Pleural, Thoracic Surgery, Video-Assisted

\section{INTRODUCTION}

Primary tumors of the pleura have been classified into 2 major categories: diffuse and solitary. Diffuse pleural neoplasms are the common asbestos-related malignant mesotheliomas arising from mesothelial tissue, while solitary tumors are not asbestos-related. ${ }^{1}$ Solitary fibrous tumors are rare neoplasms with approximately 900 cases described in the literature. ${ }^{2}$ They are known by a variety of names indicative of their clinical course and of the controversy regarding their histogenesis. Previous definitions include benign fibrous mesothelioma, localized mesothelioma, benign localized fibroma, and submesothelial fibroma; ${ }^{3}$ the preferred term for these tumors of mesenchymal origin is solitary fibrous tumor of the pleura (SFTP). ${ }^{4}$ SFTP follows a benign course in up to $80 \%$ of cases, and has no relationship to malignant mesothelioma. ${ }^{5}$ SFTP can be malignant when it is large; in this situation it presents with symptoms of compression of adjacent structures. Malignant histological aspects can also be present.
SFTP usually originates from the visceral pleura and is pedunculated, but it may arise in a wide range of tissues, which confirms its mesenchymal histogenesis. ${ }^{6}$ We describe our experience of 18 patients with a diagnosis of SFTP.

\section{PATIENTS AND METHODS}

Eighteen patients with SFTP were operated on in our institution between 1984 and 2007. Charts, radiographs, and histopathology slides were reviewed to determine the clinical presentation, histologic type, and malignant features as well as the surgical approach, operative outcome, pattern of recurrence and metastasis, and survival rate. Complete surgical resection of the neoplasm was performed in all patients, and they were all included in a strict long-term follow-up program that included clinical examination, chest radiography after 1 , 3 , and 6 months, and a computed tomography (CT) scan every year. The follow-up period ranged from 1 to 10 years; no patient was lost to follow-up.

Jury Brandolini, MD Tel: +390516363580Ｅmail: brandolini3@alice.it

Department of Thoracic Surgery, University of Bologna, S. Orsola-Malpighi Hospital, Massarenti 9, 40138 Bologna (BO), Italy. doi: 10.1177/0218492309338103

(C) SAGE Publications 2009 Los Angeles, London, New Delhi and Singapore

\begin{tabular}{lll}
\hline ASIAN CARDIOVASCULAR \& THORACIC ANNALS & 378 & 2009, VOL. 17, NO. 4
\end{tabular}

Downloaded from asianannals.ctsnetjournals.org by on December 27, 2011 
Histological diagnosis was determined in hematoxylin and eosin-stained specimens visualized by light microscopy, and immunohistochemical reactions with vimentin, actin, desmin, synaptophysin, chromogranin, neurone-specific enolase, neurofilaments, LE47, S-100 protein, Bcl-2 and $\mathrm{CD} 34$, as well as by electron microscopy. All cases were analyzed by pathologists. Special "white sections" were prepared for immunohistochemical examinations using different mono- or polyclonal antibodies (keratin, vimentin, S-100, CEA, EMA, CD34, desmin, actin) to establish the differential diagnosis between SFTP and malignant mesothelioma.

\section{RESULTS}

There were 11 women and 7 men, aged 33-77 years (mean, 56 years); 9 patients were smokers. No history of exposure to asbestos was recorded. Cough in 2 patients, chest pain in 3 , and dyspnea in 2 were the most common symptoms. One patient had hemophthisis and pleural effusion. Ten patients were asymptomatic and the diagnosis was made incidentally through a chest radiograph performed for other reasons; in these cases, there was an opaque appearance in the pulmonary parenchyma or arising from the parietal or diaphragmatic pleura, or signs of a mediastinal mass. No patient presented with hypertrophic pulmonary osteoarthropathy or digital clubbing, but one case of hypoglycemia was observed. The preoperative assessment included CT scan and bronchoscopic examination. The CT appearance of the lesions was of a homogeneous well-delineated focal mass in 15 cases, and a large heterogeneous mass in 3 (Figure 1). One patient had 2 masses: the second was smaller but they were both SFTP; another patient had 3 distinct masses arising from the parietal pleura. Preoperative bronchoscopy showed normal findings in 16 cases and extrinsic compression in 2. None of these patients presented with endobronchial growth. Magnetic resonance imaging was necessary in 3 cases to

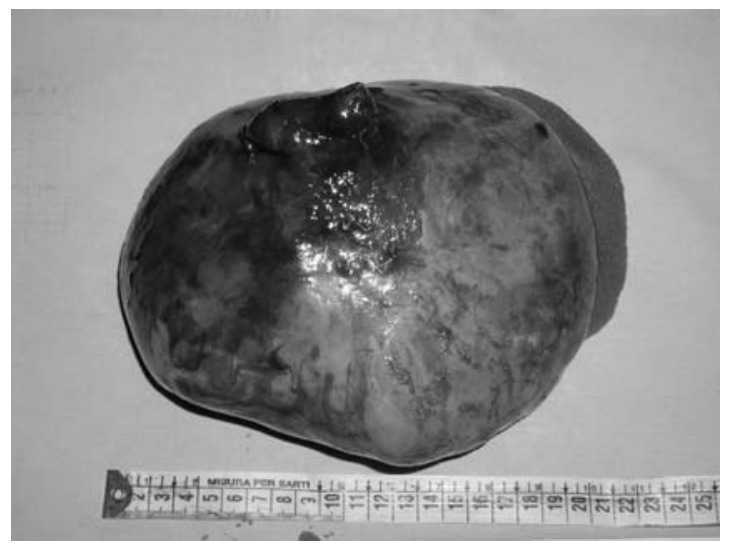

Figure 1. Computed tomography showing a giant solitary fibrous tumor of the pleura with heterogeneous zones. discriminate whether the origin of the mass was above or below the diaphragm and to determine the extent of its involvement. In 6 patients, the preoperative study was supplemented with a transthoracic needle biopsy; preoperative histologic diagnoses had been obtained in 2 of them. In 6 cases, preoperative histological diagnoses were obtained by video-assisted thoracoscopy (VAT); small lesions were removed along with the pedicles in 3 cases by video-assisted thoracic surgery (VATS) and the resections were limited. Three VAT cases were converted, so 11 patients had a thoracotomy (we performed a double thoracotomy in one), and one had a median sternotomy. All patients underwent tumor exeresis. The following operations were performed: 7 wedge resections of the left lung, one of which required partial diaphragmatic resection; 1 upper left lung lobectomy; 8 wedge resections of the right lung; and 6 masses were removed via a limited pleurectomy of the mediastinal pleura.

Tumor size ranged from 1.3 to $24 \mathrm{~cm}$ in diameter (mean, $18.8 \mathrm{~cm}$ ). The largest tumor, weighing $3,100 \mathrm{~g}$, presented with fibrohistiocytoma-like malignant features (Figure 2; Table 1). The tumors originated from the

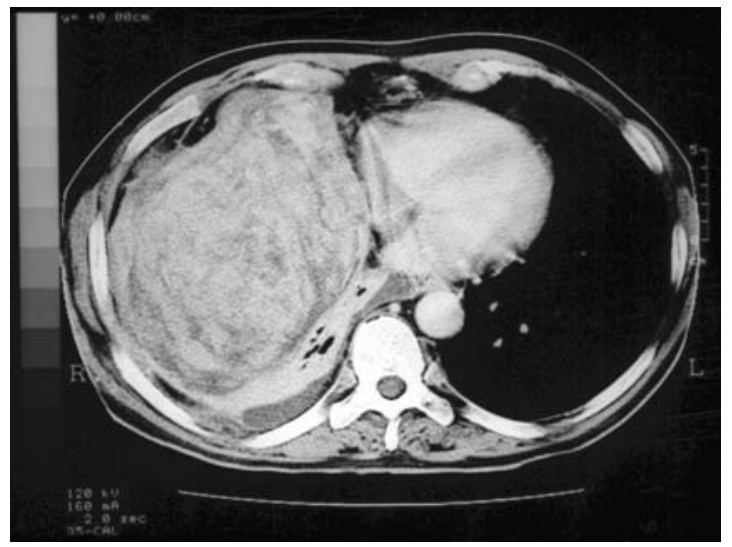

Figure 2. Giant fibrous tumor of the pleura: macroscopic specimen.

Table 1. Most frequent pathological features of small and large solitary fibrous tumors of the pleura

\begin{tabular}{ll}
\hline Small Tumors $(<8 \mathrm{~cm})$ & Large Tumors $(>8 \mathrm{~cm})$ \\
\hline Nodular within lung & $\begin{array}{c}\text { Pedunculated within } \\
\text { pleural cavity }\end{array}$ \\
Visceral pleural origin & $\begin{array}{l}\text { Parietal pleural origin } \\
\text { Smooth pleural surface }\end{array}$ \\
Uniformly solid hyalinized & $\begin{array}{c}\text { Focally edematous, } \\
\text { hemorrhagic, necrotic }\end{array}$ \\
Poorly vascular & Richly vascular \\
No mitoses & Rare mitosis \\
No pleomorphism & Variable pleomorphism
\end{tabular}


visceral pleura in 15 cases; there was a pedicle present in 8 of them, whereas 5 had no pedicle (sessile tumor) and 2 showed a prevalent intrapulmonary growth (the so-called inverted fibroma). The lesions originated from the parietal pleura in 3 cases, from the mediastinal pleura in one, from the diaphragmatic pleura in one, and from the mediastinal and diaphragmatic pleura in another. In 11 cases, the tumors had a vascular pleural pedicle ranging from 1 to $2 \mathrm{~cm}$ in length. Tumors with pedicles are always benign; however, one case of SFTP and fibrohistiocytoma had a $1-\mathrm{cm}$ long stalk. The remaining tumors were broad-based with sessile attachment to the pleura, or involvement with the pulmonary parenchyma. All neoplasms were well circumscribed, encapsulated by a thin translucent glistening membrane containing a reticulated vascular network, round or ovoid, and firm to rubbery. Only one tumor presented with a malignant component with a soft and mucoid consistency (coexistent with hemorrhage and necrosis clearly observable macroscopically). Of the 15 patients with benign tumors, 14 were free of disease after surgery. There were 3 deaths: one after 4 years due to cardiac problems, and another after 2 years from nontumor-related causes. The patient with a malignant form of SFTP died 18 months after surgery due to respiratory failure caused by pleural effusion. The 2 patients with borderline tumors (with some malignant microscopical features such as increasing mitotic activity and presence of necrosis) were free of disease at 15 and 20 months after surgery. Survival rates at 3, 5, and 10 years were $86.7 \%, 75 \%$, and $66.7 \%$, respectively.

\section{DISCUSSION}

SFTP has been described as occurring mostly in the pleura, but it has also been reported in other thoracic areas (pericardium, mediastinum), the abdomen (peritoneum, retroperitoneum, liver), and exceptionally in the nasal cavity, sinuses, nasopharynx, parapharyngeal spaces, epiglottis, thyroid, parotid, and orbit. ${ }^{3}$ SFTP may be asymptomatic for a long time, and some tumors are only recognized incidentally. The most common symptom reported is cough. ${ }^{2}$ The incidence of SFTP is approximately 2.8 cases per 100,000 hospital patients, accounting for fewer than $5 \%$ of all neoplasms involving the pleura. ${ }^{6}$

In our experience, the majority of SFTP remained asymptomatic for a long time, usually being an incidental finding on chest radiography, while symptomatic patients complained of nonspecific thoracic symptoms such as cough, chest pain, and dyspnea. Sometimes, SFTP is accompanied by extrathoracic manifestations that include arthritic pain and clubbing of the fingers (hypertrophic pulmonary osteoarthropathy) and hypoglycemia. ${ }^{7,8}$ Hypoglycemia is caused by tumor production of insulin-like growth factor I or II. ${ }^{9-11}$ The occurrence of hypoglycemia with an intrathoracic tumor was reported independently by both Doege and Potter in 1930; since then, the presence of a non-pancreatic tumor associated with low blood sugar levels has often been referred to as Doege-Potter syndrome. ${ }^{12}$ The key to diagnosis of SFTP remains chest radiography which shows a wide variation in appearance. In our cases, as in most of those described in the literature, it appears as a well-delineated, round, ovoid, or lobulated mass. If the lesion is pedunculated, the position of the mass can change, which should be indicative of the diagnosis. Serous pleural effusion affects fewer than $10 \%$ of patients, and calcification is present in the tumor in $7 \%$.

The CT scan is an important step in the preoperative work-up because it can determine the relationship of the tumor to neighboring structures and assess its resectability. CT usually reveals a sharply delineated and sometimes lobulated mass, with the same density as the musculature, which is homogeneous in most cases, especially when the lesion is small. Larger neoplasms may have a heterogeneous appearance. Contrastenhancement is usually intense and homogeneous, as a result of the rich vascularization. However, CT also may show non-enhanced areas that are due to necrosis, myxoid degeneration, or hemorrhage within the tumor. The angles between the tumor and the chest wall can be acute and suggest a parenchymal mass. When the tumor is close to the mediastinum or diaphragm, its relationship to the surrounding structures is sometimes difficult to assess, even using CT. Magnetic resonance imaging is the most sensitive examination to exclude invasion of neighboring structures. Ultrasound can help to precisely locate the tumor in relation to the diaphragm, and we used this method for confirmation of a magnetic resonance imaging result in one case. Fine-needle transthoracic aspiration cytology is generally a poor diagnostic tool because the tumor is composed of acellular and hypercellular portions. Bronchoscopy does not help in preoperative diagnosis because SFTP is an extrabronchial disease. None of our patients presented with endobronchial signs of compression, and only extrinsic compression could be seen. VAT is the most invasive method used for diagnosis. It has the highest yield (95\%) in specific and neoplastic pleural diseases. We obtained the SFTP diagnosis by VAT in 6 cases in our series.

Although SFTP is generally a benign lesion, the surgical technique must take into account the possibility of recurrence, and particularly for larger masses, the possibility of malignant transformation. In all cases, resection must not be too extensive but must ensure the absence of subsequent recurrence. In our opinion, if a 
recurrence is found in the same place as the original tumor, the new lesion and the surrounding pleura, including both the visceral and parietal pleura in contact with the tumor, should be resected. Pulmonary wedge resection by VATS is indicated for both diagnosis and treatment of a pedunculated SFTP arising from the visceral pleura. If the SFTP originates from the parietal pleura, extrapleural resection is recommended. The surgical option depends on the pleural location, the presence or absence of a pedicle, and any malignant component of the mass (such as hemangiopericytoma features). When the SFTP arises from the parietal pleura, surgical treatment usually consists of excision of the mass with its pedicle and a partial pleurectomy, but wedge resection of the pulmonary parenchyma has to be performed when the lesion arises from the visceral pleura.

Although SFTP is considered histologically benign, because of the risk of recurrence and malignant transformation, we believe that complete surgical resection should be the gold-standard treatment, with optimal disease-free survival and acceptable operative risk. VAT or VATS procedures for SFTP may obtain the diagnosis as well as complete resection of small lesions, but it is important to understand that the traditional surgical access by thoracotomy is preferable for the treatment of large masses. The duration of surgery is reduced and postoperative thoracic pain is less after VAT/VATS than after thoracotomy. Careful long-term clinical follow-up is therefore required for all cases of SFTP, and it is probably unwise to regard any such lesion as definitely benign.

\section{REFERENCES}

1. Qua JC, Rao UN, Takita H. Malignant pleural mesothelioma a clinicopathological study. J Surg Oncol 1993;54:47-50.

2. Sung SH, Chang JW, Kim J, Lee KS, Han J, Park SI. Solitary fibrous tumors of the pleura: surgical outcome and clinical course. Ann Thorac Surg 2005;79:303-7.

3. de Perrot M, Fischer S, Bründler MA, Sekine Y, Keshavjee S. Solitary fibrous tumors of the pleura. Ann Thorac Surg 2002;74:285-93.

4. Hiraoka K, Morikawa T, Ohbuchi T, Katoh H. Solitary fibrous tumors of the pleura: clinicopathological and immunohistochemical examination. Interact Cardiovasc Thorac Surg 2003;2:61-4.

5. Robinson LA. Solitary fibrous tumor of the pleura. Cancer Control 2006;13:264-9.

6. Akman C, Cetinkaya S, Ulus S, Kaynak K, Oz B. Pedunculated localized fibrous tumor of the pleura presenting as a moving chest mass. South Med J 2005;98:486-8.

7. Briselli M, Mark EJ, Dickersin GR. Solitary fibrous tumors of the pleura eight new cases and review of 360 cases in the literature. Cancer 1981;47:2678-89.

8. Balduyck B, Lauwers P, Govaert K, Hendriks J, De Maeseneer M, Van Schil P. Solitary fibrous tumor of the pleura with associated hypoglycemia: Doege-Potter syndrome: a case report. J Thorac Oncol 2006;1:588-90.

9. Adhami N, Ahmed R, Lento PA, Shimshi M, Herman SD, Teirstein AS. Fibrous pleural tumor with hypoglycemia: case study. Mt Sinai J Med 2004;71:344-6.

10. Strom EH, Skjorten F, Aarseth LB, Haug E. Solitary fibrous tumor of the pleura. An immunohistochemical, electron microscopic and tissue culture study of a tumor producing insulin-like growth factor I in a patient with hypoglycemia. Pathol Res Pract 1991;187:109-13.

11. Chaugle H, Parchment C, Grotte GJ, Keenan DJ. Hypoglycaemia associated with a solitary fibrous tumour of the pleura. Eur $\mathrm{J}$ Cardiothorac Surg 1999;15:84-6.

12. Roy TM, Burns MV, Overly DJ, Curd BT. Solitary fibrous tumor of the pleura with hypoglycemia: the Doege-Potter syndrome. J Ky Med Assoc 1992;90:557-60. 
Solitary Fibrous Tumor of the Pleura: Surgery and Clinical Course in 18 Cases Alessandro Bini, Jury Brandolini, Fabio Davoli, Giampiero Dolci, Francesco Sellitri and Franco Stella

Asian Cardiovasc Thorac Ann 2009; 17:378-381

DOI: $10.1177 / 0218492309338103$

This information is current as of December 27, 2011

Updated Information \& Services

References

Permissions \& Licensing

Reprints including high-resolution figures, can be found at: http://asianannals.ctsnetjournals.org/cgi/content/full/17/4/378

This article cites 12 articles, 4 of which you can access for free at: http://asianannals.ctsnetjournals.org/cgi/content/full/17/4/378\#BIB L

Requests to reproduce this article in parts (figures, tables) or in its entirety should be submitted via email to: info@asiapex.com

For ordering reprints, please email: info@asiapex.com

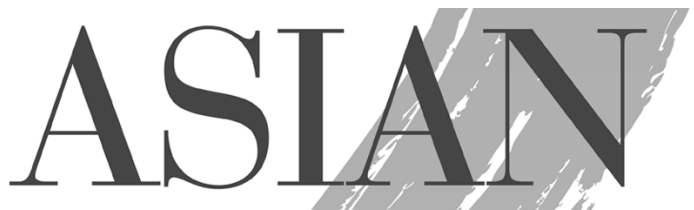

CARDIOVASCULAR \& THORACIC

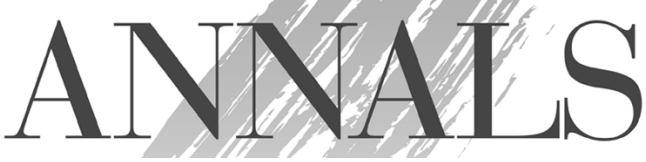

